\title{
Online Pharmaceutical Management System
}

\author{
Onuiri Ernest E., \\ Oyebanji Inalegwu G., \\ Fayehun Solomon A., \\ Chukwujioke Sam-David \\ Department of Computer Science, Babcock University, \\ Ilishan-Remo, Ogun State; Nigeria
}

doi: 10.19044/esj.2016.v12n12p139 URL:http://dx.doi.org/10.19044/esj.2016.v12n12p139

\begin{abstract}
Pharmaceutical practices have evolved over time to become fully encompassed in all aspects of pharmacy itself. Such practices include: dispensing of drugs, consultation, drug regulation, and the sale of these drugs. The community pharmacies and hospital pharmacies have key roles to play in the pharmaceutical practices. For the community pharmacies in SubSaharan Africa, a strict approach to the sale and dispensation of drugs is not normally the case, due to the fact that laws governing pharmaceutical practices have not been fully enforced. On the part of the hospital pharmacies, there is a more controlled approach to the dispensing of drugs, as the prescriptions are readily available from the in-house doctors. There is a need for these practices to be fully enforced, and a management system introduced to the fray. With software such as the Online Pharmaceutical Management System, which provides a platform has been provided to help with drug regulation, as well as providing ease to all parties involved. The methodology used in the implementation of the software is the Incremental Model of System Development Life Cycle, which allows room for scalability as time goes on. Creating an Online Pharmaceutical Management System would help in pharmaceutical practices for all parties involved. It is eminent that the system provides a safe, secure and verified platform for all parties which help to bridge the communication gap and provide legitimate drugs. Therefore, if all recommendations are strictly adhered to, there will be strict monitoring and regulation of how drugs are circulated and a decrease in the spread of fake drugs.
\end{abstract}

Keywords: Pharmaceutical practices, Platform, Inventory Management, Hospital Pharmacy, Community Pharmacy, Drug regulation 


\section{Introduction}

The practice of pharmacy involves the general implementation of medical orders which entails the evaluation and the interpretation of the medical orders, the administration of drugs, dispensation of prescribed drugs from qualified medical practitioners, the review of prescribed drug regimen, and the correct storage of drugs (CPP, 2009). The American Pharmacists Association has described pharmacy as having a mission which is the responsibility of ensuring the use of medication in the right way and manner, and enjoying the services of drugs to ensure the achievement of optimized results in therapeutic applications (CPP, 2009).

In addition, the birth of modern pharmacy has brought with it some positive effects in the society; like the increase in life expectancy globally from 45 years to 50 years, which some argue is as a result of the number of breakthroughs recorded in pharmacy over the last century. These breakthroughs such as: the discovery of new compounds in battling existing ailments and the invention of methods and devices to accurately dispense drugs have been welcomed with open arms by the society. (Federation Internationale Pharmaceutique, 2012).

More so, the hospital pharmacy is usually located in hospitals (government-owned or private) and provides in-house pharmaceutical care to patients. Hospital pharmacists are those that function within a hospital pharmacy system and are tasked with the dispensing of prescriptions, the purchase of medicines, and the testing of these medicines (World Health Organization, 1994). In the hospital pharmacy, access to medical records make it easy to influence the selection of drugs based on allergies or previous problems encountered. Subsequently, the power to control the process of going into the acquisition of drugs and other pharmaceutical devices, help in ensuring the supply of premium quality drugs. Furthermore, Hospital pharmacies which are owned by the government have little or no problems with financing projects due to backing from the government (El Tayeb, n.d.).

Also, the community pharmacies may not have the permission to dispense certain drugs which may be due to the nature of such drugs and the prices of drugs are not as subsidised as those in hospital pharmacies (Postnikoff, n.d.).

The proposed system aims to connect and serve as an intermediary between hospital pharmacies and drug manufacturers, through a platform that allows the order of drugs with the click of a button, and simultaneously keeps track of what drugs have been ordered and in what quantity. 


\section{Review of closely related works}

Taking a look at Sub-Saharan Africa in general, the online pharmaceutical field is relatively underdeveloped and an untapped one. The available systems have not fully captured the essence of a pharmaceutical inventory system. In view of the foregoing, this research work aims to rectify this problem by providing the platform for the ever-growing pharmaceutical industry in the country, and region as a whole.

\section{Content management systems}

A Content Management System (CMS) is one that is used to manage information, be it web-based systems or information delivery systems. These kinds of systems carefully manage data in an organized fashion in documents and borders that can be easily understood and is also presentable (Holm, Rudis \& Wilson, 2015). Content management has been around for a while, but did not eventually become acceptable till the age of technology such as the Internet, for example, or the portable computer (Swanson, Broekmeier, \& Anderson, 1982). The content stored in the system is done in a native format which enables the easy retreival of information. Most content management systems have various processes embedded in them during the retrieval of information. They also enable assessibility features such as search boxes, user interaction, customization, all of which improve on user experience (Rockley, Manning, \& Kostur, 2003).

CMS are contained in frameworks which are used for online systems which involve trading of goods and services. One of such services can be seen in Amazon, which is an American company that deals with the sale of all kinds of goods from verified vendors.

\section{Pharmacy inventory management systems}

The growing popularity of computers and gadgets in general has in no way slowed down the birth of new innovative ways in the pharmaceutical management system. These days, computers have become an essential part of many people's lives due to the versatility of the devices, and how much they can do with so little effort. Performing tasks which would have taken hours and maybe days in the past, have become possible in seconds. The invention of the Internet and other utilities such as search engines (Google being a prime example), have made searching for the most remote things possible in very little time. Also, the portability of the computer systems have helped the cause in making information more portable than it was in the past.

In the same vein, the management of inventory and taking stock of goods and services in different organizations have become so much easier with the growth of the computer system (Goldberg et al, 1991). These days, 
even phones and tablets have faster processing power than the early generation computers, which has made it easier to perform tasks. Our economy has been referred to as the "learning economy", because of the rate at which new innovations come to light (Abbas, Alhasan, \& Hamza, 2015). In China, a text messsage system was developed to help in general patient pharmaceutical care, and promote mobile systems (Mao, Zhang, \& Zhai, 2008).

The management of inventory has taken various shapes and forms, one of which is called a Vendor-Managed Inventory (VMI) system. The VMI gives a supplier full discretion to deliver goods and services, as long as the customer can fully optimize these goods and services. This method or level under the VMI system is called the Maximum Level. The other level in the system is called Order-up-to policy which allows the supplier to get the inventory of the customer to its maximum capacity at every delivery (Coelho \& Laporte , 2015).

Various computerized management systems have been useful in helping to save lives around the world. The earthquake in Haiti in 2010 brought about a need for medicines for the injured, and also aid for them. Due to the high influx of medicines into the country at the time, an inventory managemnt system was developed to help the cause. The hospitals in the country did not at the time have the means to provide medicines to the needy without losing track of what has been given to who, and what has not been given. Also, the management systems that were proposed and later implemented helped in tackling shortage problems, thereby saving countless lives in the process. The Pharmacy Computerized Inventory Program (PCIP) had four processes which include: needs assessment, the development of the PCIP, implementation of the PCIP, and outcomes and data analysis. The program proved to be a success and greatly reduced the turmoil going on in the land at the time of the earthquake (Holm, Rudis, \& Wilson, 2015).

Other parts of the world such as the Middle East have not been left out of the trend. A group of individuals in King Saud Bin Abdulaziz University for Health Sciences in Saudi Arabia came together to study and report findings on a pharmacy inventory management system in a hospital in Saudi Arabia. Their software controlled and monitored existing stock levels, which allowed them to decide how much quantity of drugs to order in order to be at full capacity. The application was installed on all computers of the staff in the hospital which was used and information was directly updated to the database (Muallem, Dogether, Al Assaf, Al Ateeq, \& Househ, 2015)

These various systems have allowed ease of work at the hospitals and pharmacies, and reduction of errors in drug related practices. Also, with the introduction of these practises, the prescription drug abuse trend has been 
curbed to a considerable extent. These days, applications make use of large databases and verification processes.

\section{Cvs pharmacy mobile application}

This application is used on phones and tablets and runs on the android and iOS platforms on these devices. The mobile application is used for prescription management and offers discount to users of the application.The application has proven helpful in drug prescription management as it offers a number of features. It allows its users to manage what prescription drugs they need to take and when they need to take them. The application has proven to be helpful to the older folk who are more vulnerable to diseases due to aging. The users may forget what time they need to take their prescription or the dosage to take, but the application notifies them and prevents missed dosages (CVS, 2015).

The advantages are as follows:

1. It is easy to download as the file is light is size

2. It helps users to know when to take their drugs

The limitations are as follows:

1. It lets the user order drugs which may promote addiction

2. Although the interface is fluid and fast, it may not be simple enough for users

3. The application has many bugs, such as unexpected application failure that need to be fixed, to fully optimize its use.

4. It does not show drug composition, which allows users know if there are side effects in using medicine.

\section{Walgreens mobile application}

This application allows users to scan barcodes at drug dispensaries and keeps track of medication in-takes.The application is vital in checking for the validity of drug prescriptions, as individuals have shown that prescription of drugs may be faked. The application allows users to create accounts which are linked to the barcodes of specific drugs, which allows the users of the application to have some sort of verification in the picking up of the prescription drugs (Walgreens, 2015).

The advantages are as follows:

1. It allows users to scan barcodes at dispensaries which brings a new innovation

2. It is light and easy to download and can be accessed on a phone or on other mobile devices

The limitations are as follows:

1. It is slow to respond and has bugs 
2. It allows users to be in control of what drugs they take, which may promote addiction.

3. The application does not work in many parts of the world.

\section{Pharmacy ci mobile application}

Operating from Ivory Coast in Africa, the Pharmacy CI application allows its users to access a list of pharmacies on duty at any particular time. The application is available on the iOS and Android platforms.

The advantages are as follows:

1. It is easy to use and its design is fluid

2. It gives accurate directions to its users on where the pharmacies are located.

The limitation is that it may promote drug abuse as it allows users to profile each pharmacy, to know which ones would be more acceptable in selling dangerous drugs, based on location and other factors.

\section{Methodology}

The approach that was used in the design of the system is the Incremental Model of System Development Life Cycle where the product being designed is implemented and tested incrementally. It is relatively cheap and is used for small projects. This methodology is most suitable for the project due to the project's future requirements which would require changes in the system (Muallem, Dogether, Al Assaf, Al Ateeq \& Househ, 2015). This is further broken down in the use of:

1. JavaScript and Cascading Style Sheet tools for the web page components of the application

2. PHP as a server-side scripting language to compute records and information.

3. MySQL for database management, and AJAX to bring web application features to the system.

\section{Functional requirement}

1. The admin's privileges are role based, thereby allowing for Role based access control for security

2. Each users IP address is stored in the database for security

3. Users of the system are banned after a 4 failed authentication process

4. Users can view products out of stock but cannot card them to cart

5. Each user has a unique identifier 


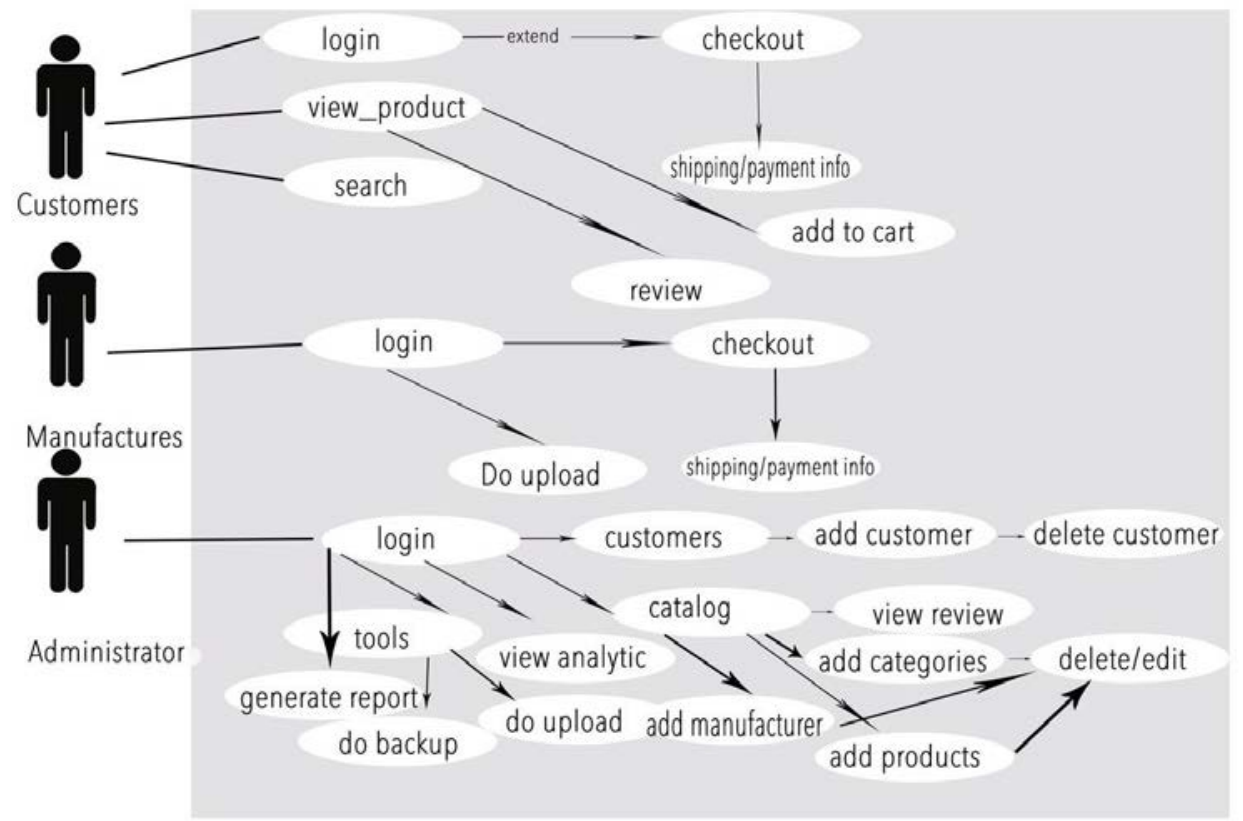

Fig 1: Use-Case Diagram

Figure 1 shows all the relationships between the actors in the system.

\section{System analysis and design}

The requirement specifications from the first phase are studied in this phase and the system design is conducted. Analysis of existing system is also carried out in this phase; the limitations of the existing system are analyzed and improved upon. 


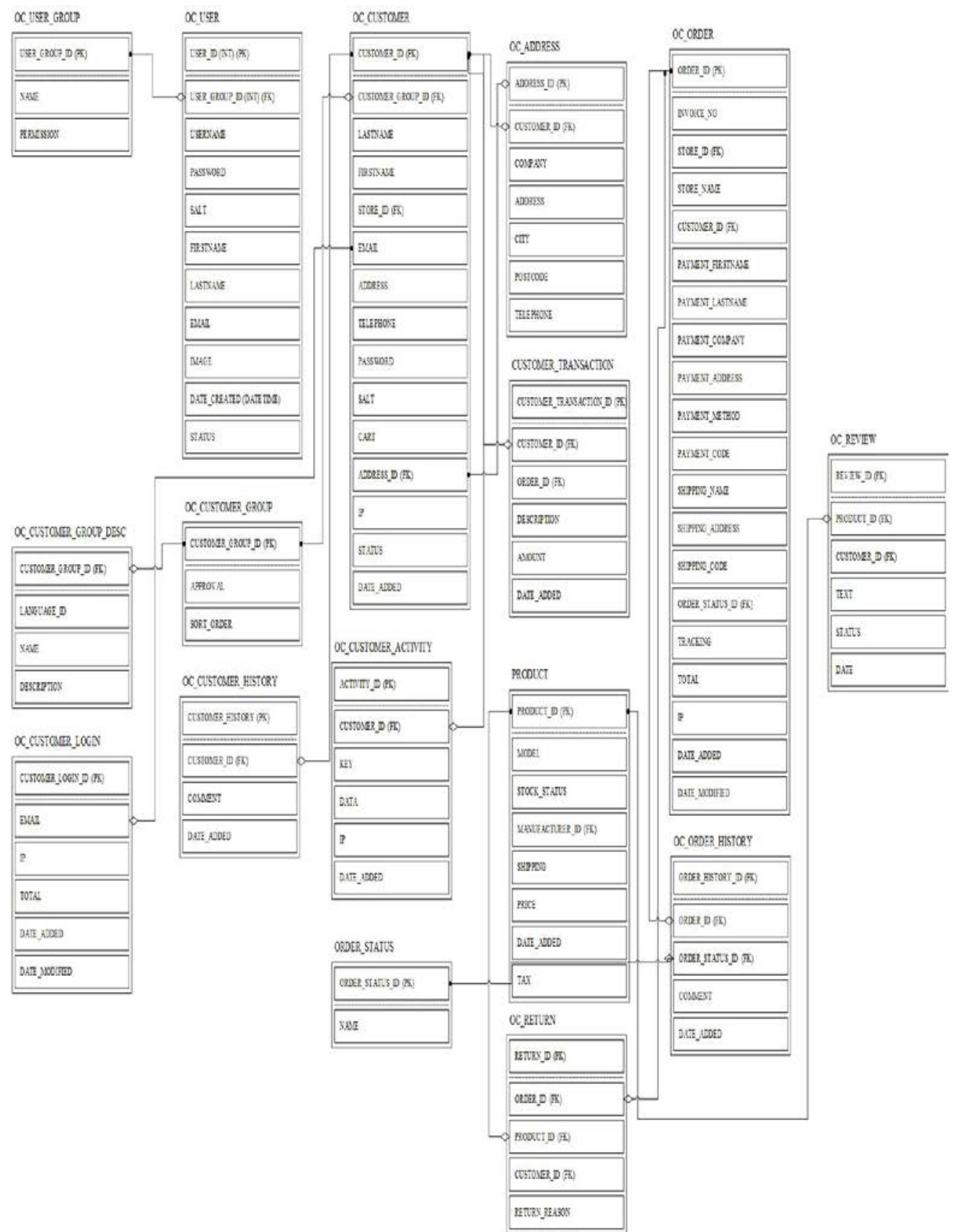

Fig 2: Entity-Relationship Diagram

Figure 2 shows the relationships between all the entities in the system

\section{Software requirements}

1. Operating system: Windows (98, 2000, ME, NT, XP, Vista, 7, 8, 10), Linux, Mac OS. 
2. JavaScript-enabled web browsers: Mozilla Firefox (most suitable), Internet Explorer, Google Chrome, and Opera-mini.

3. Virtual Server: XAMPP server

\section{Hardware requirements}

1. A physical memory (RAM) of 512MB and above are required

2. Intel, Celeron or AMD Pentium 3 processor

3. Hard disk capacity: 5 GB.

\section{System implementation and review}

In computer science, implementation is specifically categorized as the realization of a technical specification or algorithm as a program, software component through computer programming and deployment.

The system runs on a number of languages and tools which make it fully responsive and dynamic. These include:

1. PHP: It serves as the server-side scripting tool on this application. PHP allows the web application to be responsive and dynamic. What this means is that the server hosting the content can respond to user requests in different manners.

2. JavaScript: This is the client-side scripting tool on this web application. JavaScript can be used either as a server-side scripting tool or a client-side scripting tool. It promotes interactivity with the users and makes the web application more dynamic.

3. HTML: This stands for Hypertext Markup Language. It is a web standard for achieving text, colour etc. for the web application.

4. CSS: This creates a fluid design for the web application. It works hand in hand with HTML to create a user-friendly design on the web application.

5. MySQL: It is a relational database system that is designed to work with multiple systems. This tool is used to manage information in the database of both small and large systems.

The system has PHP as its base which works hand in hand with the other languages and tools to bring a dynamic experience to the Online Pharmaceutical Management System. Due to the fact that the system runs online, the use of PHP and JavaScript were vital so as to improve interactivity with the users of the system. Also, HTML and CSS help to fully encapsulate the dynamisms and interactivity of a standard web-based system.

On the system, all the products are stored in various tables in the database, for clear distinctive purposes. What the user sees is the Graphical User Interface (GUI) which allows easy access to products and services. The GUI of the system is as a result of all the tools and languages, both at the server-side and client-side, coming together to form a cohesive unit. All the 
user has to do is to provide correct login details, which is verified by the database, to access all products.

There is also an administrative dashboard which allows only the administrator to add or remove users. This feature was introduced to check for any intruders or unauthorised personnel in the system, who may want to order drugs illegally.

This system may be likened to other established web-based applications such as Amazon and E-bay which allows its users to buy products using a cart system such as the one implemented on the Online Pharmaceutical Management System.

Also, with the use of the programming languages and tools mentioned above, the system is fully adaptable to all devices available today.

\section{System testing}

System Testing can be done at two stages namely:-

1. Unit Testing, the system is tested in modules before integration is done. This is important as faults are discovered before the systems complexity increases through system integration.

2. System Testing, the system is tested for conformity with requirements after all modules have been put together and the system as a whole is tested to authenticate that general system requirements have been met.

In the development of the System, various criterions were used as testing yardsticks of the system. These yardsticks include Graphic User Interface, usability testing, database and exception handling (Martin, 1991). Both the black-box and white-box methods were used in the unit testing.

\section{Graphical user interface testing}

The Graphical User Interface (GUI) testing involves testing the systems graphic components to ensure that it covers the entire domain i.e. the complete functionality of the system, with respect to the different modules. For example, in the testing of the System, both customer service and administrator modules were tested to ensure that they all contained links and tables that directly links to their requirements as designed with use cases in the preceding section. There also comes a stage of sequencing testing with GUI testing. This ensures that domains of requirements have an efficient follow up order, that is, users are less likely going to be confused or going to find it difficult to navigate around the system in search of how to perform some sort of operation. For example, how the user can view details of a product and the manufacturer of the product. 


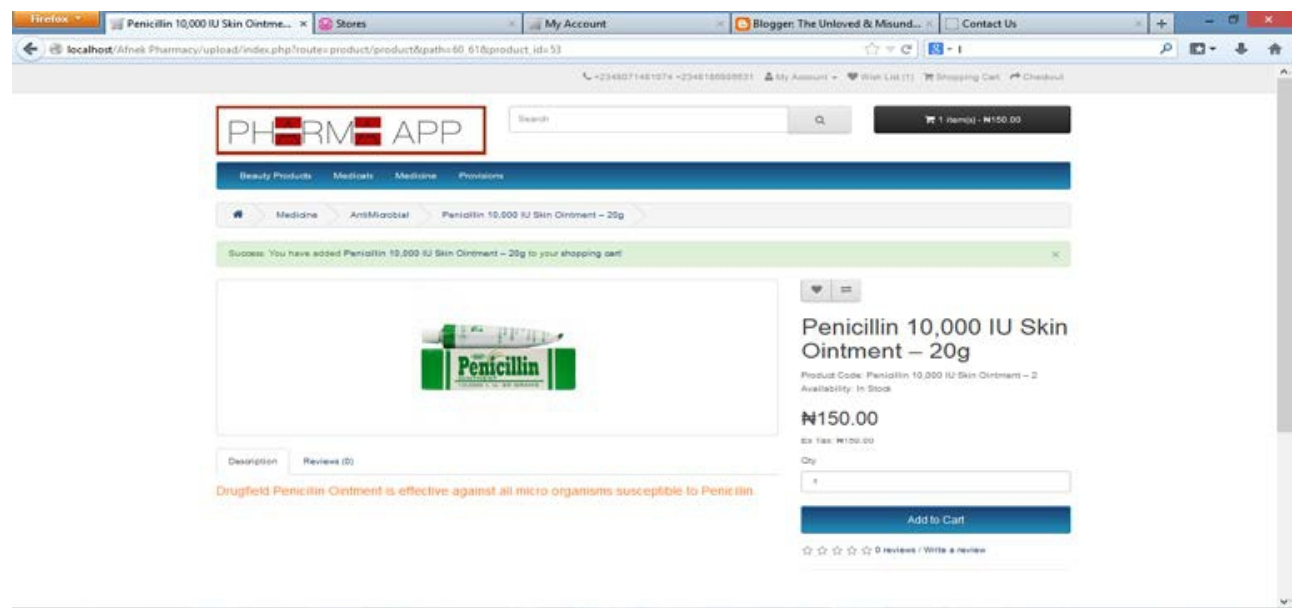

Fig 3: Adding a product to the shopping cart

Figure 4 shows how a product is added to the shopping cart of the application

\section{Usability testing}

This essentially focuses on testing systems user-friendliness. It involves testing a web application to find out directly how users communicate with the system. The test cases were developed to have been realistic enough.

For example, a few random persons can come to the site and view all the products, contact the administrators for registration, login to their account after a successful registration, contact the manufacturer. The test case was successful because the users reported ease of use of the system.

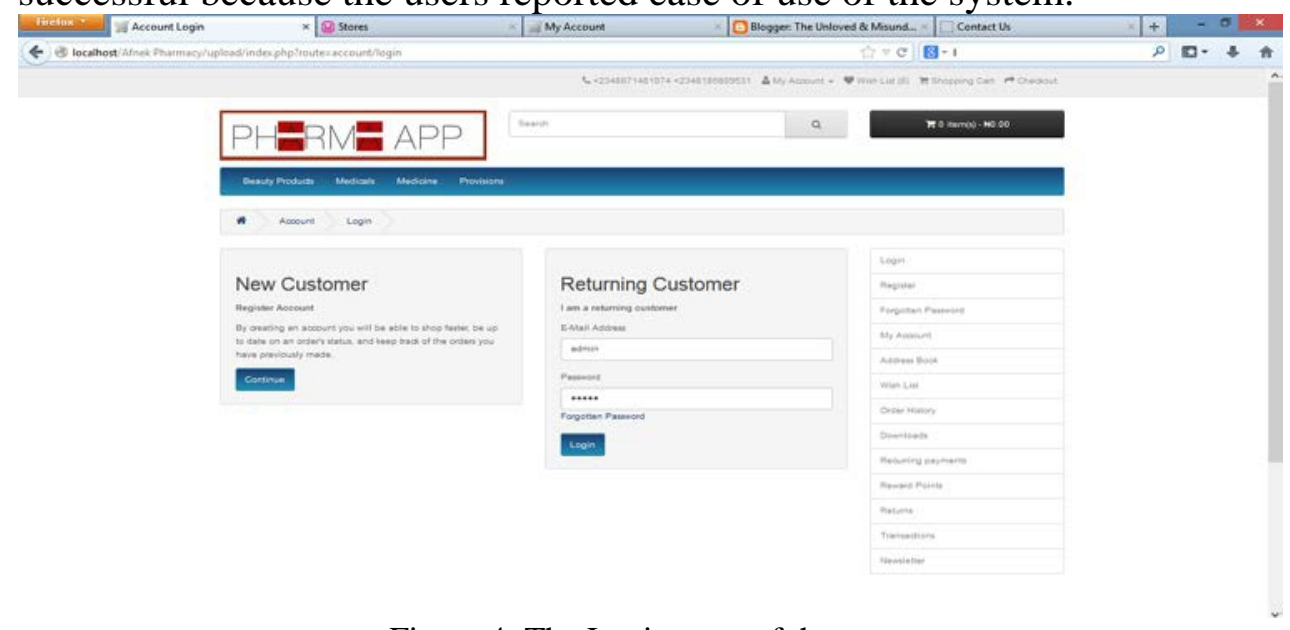

Figure 4: The Login page of the system 
Figure 4 shows the login page of the system, as well as directions on registering for the new customers.

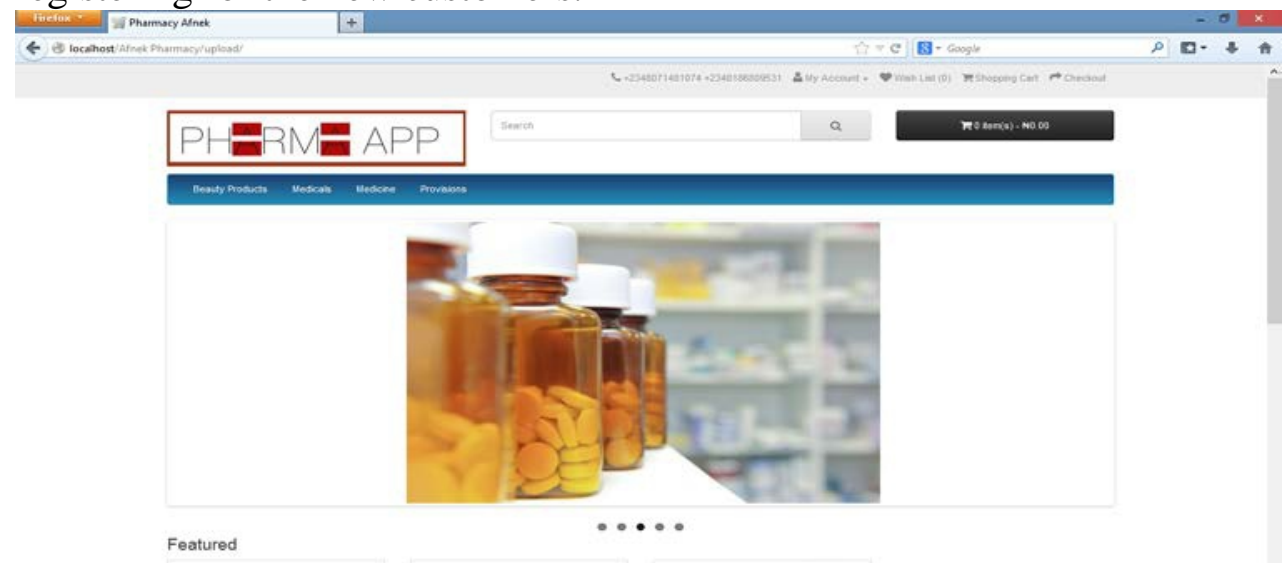

Figure 5: The homepage of the system

Figure 5 shows the homepage after a successful login attempt

\section{Exception handling testing}

Exception Handling Testing was conducted on the System to assure that the system remains viable even on the occurrence of errors as a result of anomalous or exceptional events being triggered. Various exceptions were discovered and corrected through the use of underlying JavaScript and PHP scripts. If else statements, try and catch statements in these scripts provided methods to control user actions and indirectly maintaining ordinance in the system by handling unexpected errors.

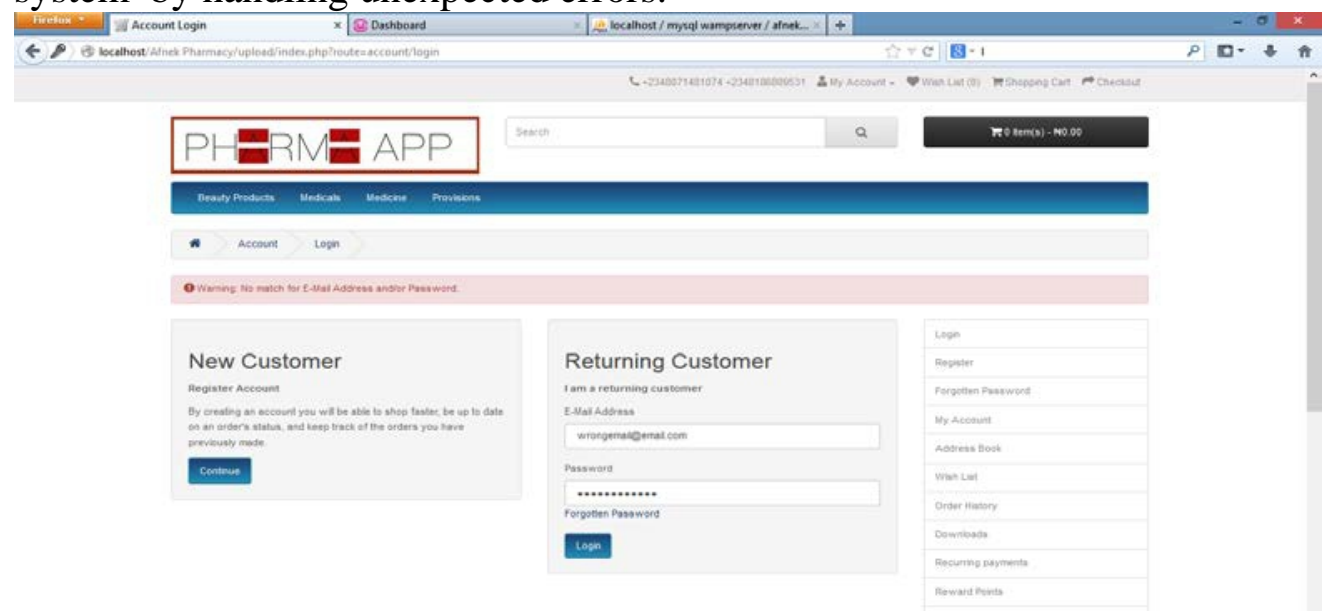

Figure 6: Login page error

Figure 6 shows an error message when a customer that has not been registered by the admin tries to login to the application. 


\section{Database testing}

Database used in the System contains a data dictionary of varying data types. These data types represent the forms of data which the data of the system can take. The data dictionary must be tested to confirm that it has been properly used to implement the system database through the Database Management System (DBMS). The database must also be tested to make sure that the system satisfies the ACID properties (Atomicity, Consistency, Isolation, and Durability) of a DBMS. Database testing was conducted on the Customer Complaints Report System with its data dictionary at hand so as to ensure that the insert, delete and update operations are as consistent as possible to avoid the possibility of occurrence of database crash as volume of data generated increases. Improper table relations were discovered and were removed such as the manufacturer's address - pharmacy address relation, and manufacturer's tax payments - pharmacy's payment options relation.

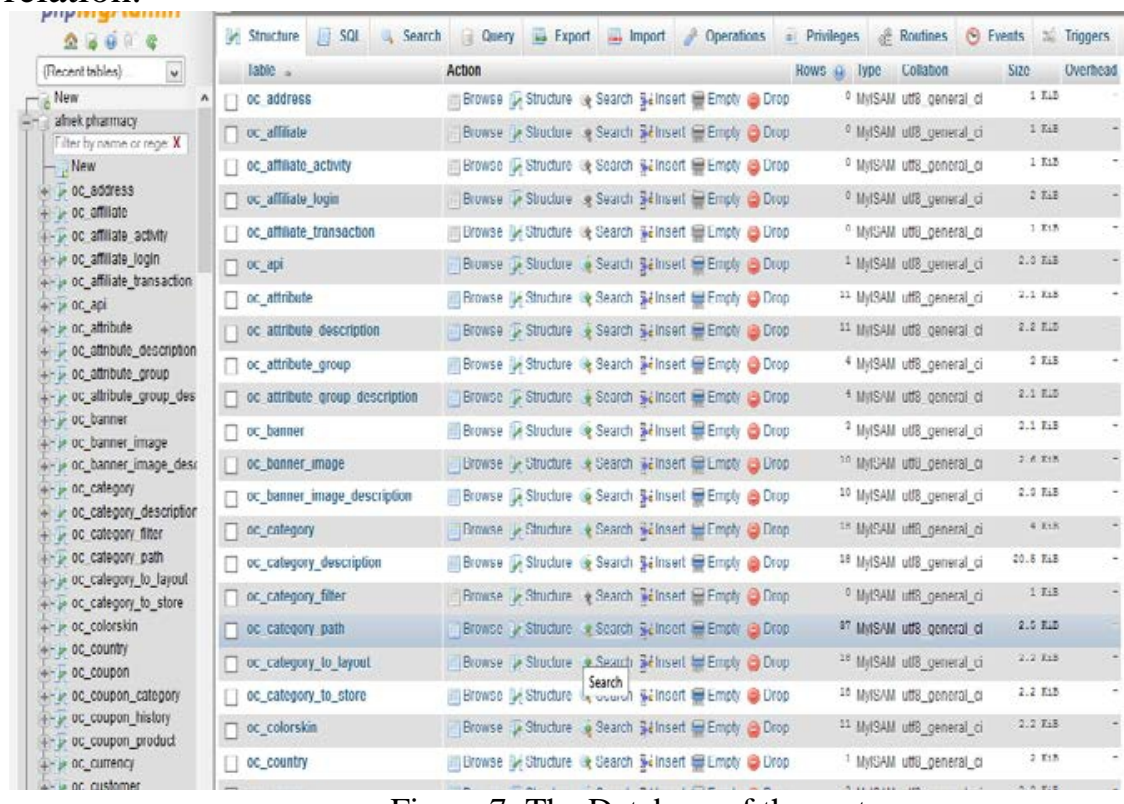

Figure 7: The Database of the system

Figure 7 shows an overview of all the tables contained in the database of the application. 


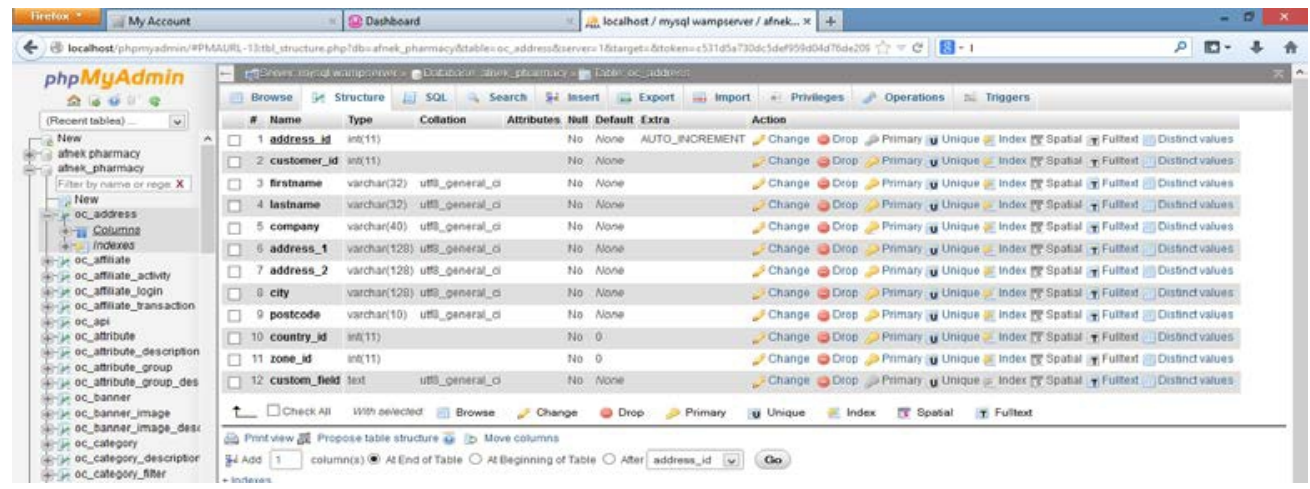

Figure 8: Pharmacy Information Table

Figure 8 shows the information table, which contains all the information about the pharmacies registered. The table keeps track of important details such as their addresses, company name, postcode, city, country, and zone.

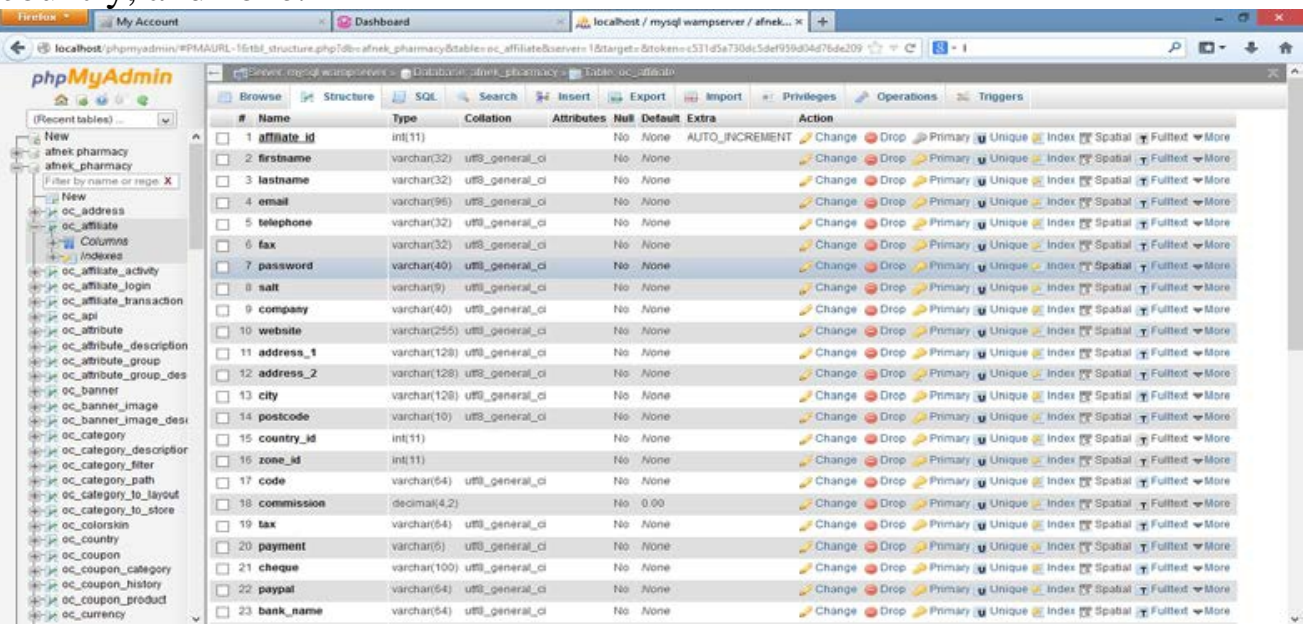

Figure 9: Manufacturer information table

Figure 9 shows all the relevant information about the manufacturers that have been registered on the system. The table contains information such as: name, email, telephone numbers, fax numbers, password, company name, website, addresses, city, postcode, country, zone, payment methods, tax documents, commission, and account numbers,.

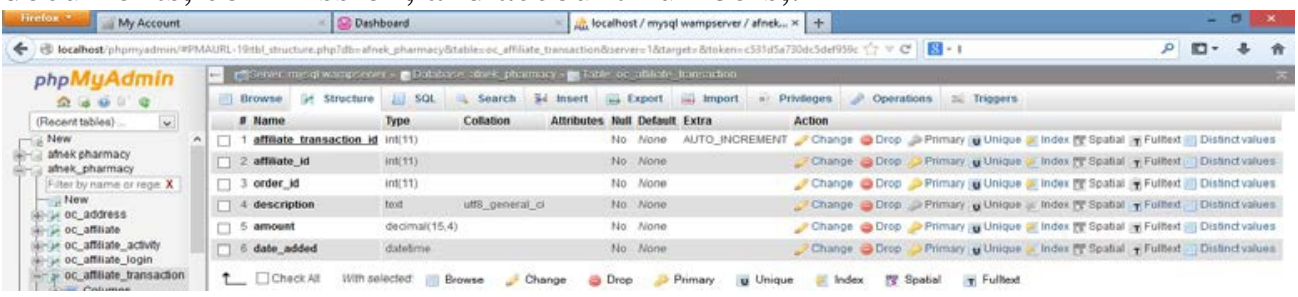

Figure 10: Transaction table 
Figure 10 shows the transaction table which contains information such as affiliate_transaction_id, affiliate_id, order_id, description, amount, and date_added.

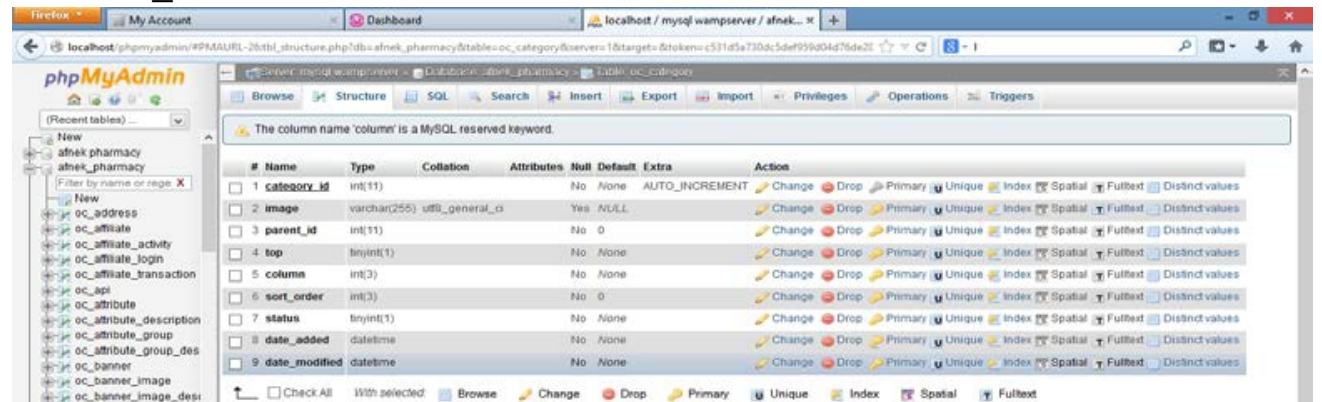

Figure 11: Category Table

Figure 11 shows the category table. In this table, the descriptions of the products being offered are stored.

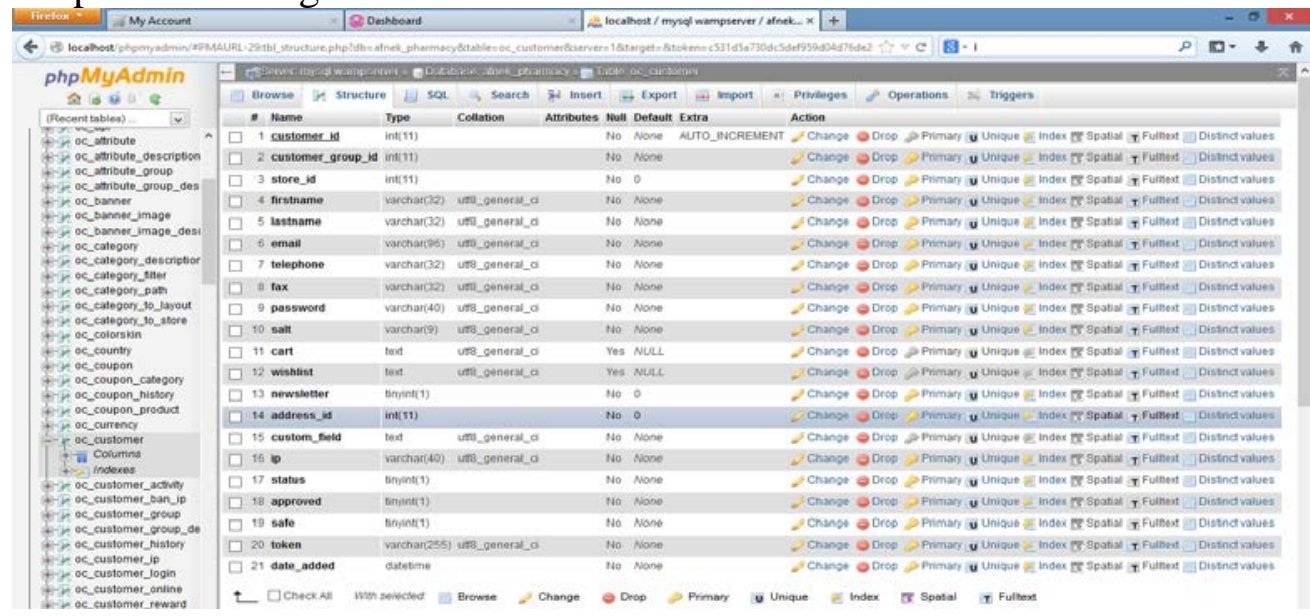

Figure 12: Customer table

Figure 12 shows a database table which contains vital information about the customer. Information in this table enables the customer to purchase the products available.

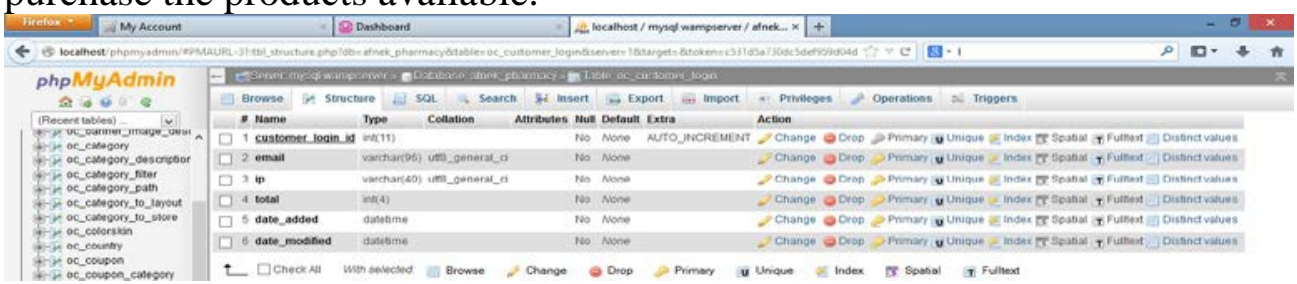

Figure 13: Customer login table 
Figure 13 contains information on the customer such as customer login details, IP address, login id, email address, date added, and date modified.

\section{Results/findings}

The Online Pharmaceutical Management System in its design aimed to limit individual personal usage. Being that the application is designed for pharmacies as a whole, as opposed to being tailored for individual use, the issue of abuse of drugs has already been diminished with the use of the application. Furthermore, only authorized personnel on the application are able to make the order of drugs from the drug manufacturers. All transactions made are stored in the system to allow record keeping. Findings made from this work show that the awareness and sensitisation to the availability (conceptual or otherwise) of online pharmaceutical applications is minimal. Also, the use of these available applications (as discussed in the closely related works section) is also minimal.

The administrator's dashboard allows only the administrator add users on the system. All data are submitted to the administrator, along with all the relevant authentic documents. This feature prevents unauthorised users from ordering the drugs available, and also controls the accounts available.

\section{Recommendations}

In view of the challenges associated with Online Pharmaceutical Systems, the researcher makes the following recommendations:

1. All the stakeholders must be computer literate and own a smartphone because the system is hosted online

2. Awareness of the Online Pharmaceutical System should be made to sensitize people about the significance of the system

3. The various regulatory bodies of the Pharmaceutical Industry should incorporate the use of the system into their regulations.

4. Benchmark research must be carried out by researchers to evaluate the performance of the system

\section{Limitations}

A number of limitations were encountered in the course of preparing this research work. One of such was in the creation of the tables in the database of the system. Due to the size of the system, many tables had to be created to accommodate all the data required in the management system. Also, implementing security features on the system proved to be a challenge as the application is yet to be tested on a national/regional level. 


\section{Future research}

Due to the findings from carrying out this research on Online Pharmaceutical Management System, some points to consider in its implementation in the future include:

1. A platform capable of use at a regional/national level

2. Strict security measures put in place to prevent an abuse of the application in general

\section{Conclusion}

The management system employs strict measures to protect the users from intruders or outsiders. One of such measures is the inability of a prospective user to register on the platform without the permission of the administrators. To register on the platform, all necessary details such as: name, address, tax documents, payment methods, and relevant licenses are sent to the administrator for proper verification. When the authenticity of these documents have been proven, the administrator then creates the account for the customer and forwards the details for login to the management system. Also, since the drugs purchased through the platform are for pharmaceutical purposes, and as such, are ordered in bulk, if a small quantity of drugs is ordered, the administrators are notified, who then confirms the order from the pharmacy to ensure that an intruder has not obtained their login details.

This research work dealt with Online Pharmaceutical Management Systems. It is eminent that the system provides a safe, secure and verified platform for all parties which help to bridge the communication gap and provide legitimate drugs. Because drugs are harmful when abused or misused by individuals or organizations, security checks have been added to the design logic. Therefore, if all recommendations are strictly adhered to, there will be strict monitoring and regulation of how drugs are circulated and a decrease in the spread of fake drugs.

\section{References:}

Abbas, M., Alhasan, A., \& Hamza, U. M. (2015). Perceived Ease of Use and Utilization of E-Learning Technologies by Academic Staff in Federal College of Education, Zaria

Coelho, L. C., \& Laporte , G. (2015). Vendor Management Systems.

CPP. (2009). Scope of Contemporary Pharmacy Practice: Roles, Responsibilities, and Functions of Pharmacists and Pharmacy Technicians CVS, P. (2015). CVS/pharmacy Unveils New Look and Enhanced Features for its Top-Rated Mobile App. Retrieved from prnewswire.com: www.prnewswire.com 
ElTayeb, W. A. (n.d.). Hospital Pharmacy. Retrieved from www.alawi.weebly.com

Federation Internationale Pharmaceutique. (2012). From Making Medicine to Optimising Outcomes: The evolution of a profession 1912-2012.

Goldberg, D. E., Baardsgaard, G., Johnson, M. T., Jolowsky, C. M., Shepherd, M., \& Peterson, C. D. (1991). Computer Based Program for Identifying Medication orders requiring Dosage Modification Based on Renal Function

Holm, M. R., Rudis, M. I., \& Wilson, J. W. (2015). Medication supply chain management through implementation of a hospital pharmacy computerized inventory program in Haiti.

Mao, Y., Zhang, Y., \& Zhai, S. (2008). Mobile Text Messaging for Pharmaceutical Care in a Hospital in China.

Martin, J. (1991). Rapid Application Development. Macmillan

Muallem , Y., Dogether , M., Al Assaf, R., Al Ateeq, A., \& Househ, M. (2015). A Pharmacy Inventory System in Saud Arabia: A Case Study .

Postnikoff, L. (n.d.). Prescription Dug Abuse- Pharmacists Role. Retrieved from www.scp.in1touch.org

Rockley, A., Kostur, P., Manning, S. (2003). Managing Enterprise Content: A Unified Content Strategy.

Swanson, D. S., Broekmeier, R. L., \& Anderson, M. W. (1982). Hospital Pharmacy Computer Systems .

Walgreens. (2015). The Walgreens App Makes Life Easier.

World Health Organization. (1994). The Role of the Pharmacist in the Health Care System. 